Supporting Information:

\title{
Quantum Mechanics/Molecular Mechanics modeling of drug metabolism: Mexiletine N-hydroxylation by cytochrome P450 1A2
}

Richard Lonsdale, ${ }^{\dagger},{ }^{\top}$ Rachel M. Fort, ${ }^{\dagger}$ Patrik Rydberg, ${ }^{\ddagger}$ Jeremy N. Harvey, ${ }^{\dagger} \S$ and Adrian J. Mulholland ${ }^{*, \dagger}$

${ }^{\dagger}$ Centre for Computational Chemistry, School of Chemistry, University of Bristol, Cantock's Close, Bristol, BS8 1TS, UK. "Department of Drug Design and Pharmacology, Faculty of Health and Medical Sciences, University of Copenhagen, Universitetsparken 2, DK-2100, Copenhagen, Denmark "Current address: Oncology iMed, AstraZeneca, Cambridge CB4 OFZ, UK. ${ }^{\circledR}$ Current address: Quantum Chemistry and Physical Chemistry Section, Department of Chemistry, University of Leuven (KU Leuven), Celestijnenlaan 200F - box 2404, Leuven, Belgium

\section{CONTENTS}

Part A: Details of initial structure preparation and molecular dynamics simulations.

Part B: Interatomic distances calculated during molecular dynamics simulations.

Part C: QM/MM energies of all species (BSI)

Part D: QM/MM energies of all species (BSII)

Part E: QM/MM energies calculated with the BHandHLYP and BP86 density functionals

Part F: QM geometries of stationary points for profile B1 


\section{Part A: Details of initial structure preparation and molecular dynamics simulations.}

The initial enzyme structure used in the MD simulations was built from a single crystallographic structure of CYP1A2, (PDB ID 2HI4, $1.95 \AA$ resolution). GOLD (Genetic Optimization for Ligand Docking) version 3.2 was used to calculate a number of initial docking poses for the mexiletine within the active site of the enzyme. The CHEMSCORE scoring function specially parameterized for hemecontaining proteins was used. ${ }^{1}$ The GOLD program has been found to successfully predict the binding positions of substrates for CYP1A2. Four of these docking poses were selected for preparation for MD simulations based on their suitability. This suitability was assessed as a combination of docking score and proximity of the mexiletine $\mathrm{NH}_{2}$ moiety to the heme iron.

All MD simulations were performed with stochastic boundary conditions, using a well established and tested protocol. The HBUILD function of the CHARMM program $(\mathrm{v} 36 \mathrm{~b} 2)^{2}$ was used to add hydrogen atoms to each protein-ligand complex according to their pKa values as calculated by the PROPKA program ${ }^{3}$. All MD simulations and MM calculations were carried out using the CHARMM27 force field ${ }^{4}$. In order to convert the protein to the active catalytic species, Cpd I, an oxygen atom was added to the heme group; this was achieved by superimposing the structure with the heme group of the $\mathrm{P}^{4} 50_{\text {cam }}$ crystal structure ${ }^{5}$, which has an oxygen atom bound to the iron. The system was then truncated to a sphere of radius $25 \AA$, centered on the heme iron atom. Neutral patches were applied for arginine, glutamate, lysine and asparagine residues near the surface of the protein, where the charged regions of the side chains were further than $20 \AA$ from the heme iron. These patched residues have the same geometry as normal residues, but have neutral charge in order to avoid unrealistic effects that could arise from cropping the protein and the

${ }^{1}$ Kirton, S. B., Murray, C. W., Verdonk, M. L., Taylor, R. D. (2005) Prediction of binding modes for ligands in the cytochromes P450 and other heme-containing proteins", Proteins, 58, 836-844.

${ }^{2}$ Brooks, B. R., Brooks III, C. L., Mackerell, A. D., Nilsson, L., Petrella, R. J., Roux, B., Won, Y., Archontis, G., Bartels, C., Boresch, S., Caflisch, A., Caves, L., Cui, Q., Dinner, A. R., Feig, M., Fischer, S., Gao, J., Hodoscek, M., Im, W., Kuczera, K., Lazaridis, T., Ma, J., Ovchinnikov, V., Paci, E., Pastor, R. W., Post, C. B., Pu, J. Z., Schaefer, M., Tidor, B., Venable, R. M., Woodcock, H. L., Wu, X., Yang, W., York, D.M. and Karplus, M. (2009) "CHARMM: The Biomolecular simulation Program J. Comput. Chem., , 30, 1545-1615.

${ }^{3}$ Li, H., Robertson, A. D., and Jensen, J. H. (2005) Very Fast Empirical Prediction and Interpretation of Protein pKa Values. Proteins, 61, 704-721.

${ }^{4}$ MacKerell, A. D., Banavali, N., and Foloppe, N. (2001) Development and current status of the CHARMM force field for nucleic acids. Biopolymers, 56, 257-265.

${ }^{5}$ PDB accession ID 1DZ9; Schlichting, I., Berendzen, J., Chu, K., Stock, A. M., Maves, S. A., Benson, D. E., Sweet, R. M., Ringe, D., Petsko, G. A., Sligar, S. G. (2000) The catalytic pathway of cytochrome p450cam at atomic resolution. Science, 287, 1615-1622. 
subsequent reduced screening of charges by solvent molecules. This led to an overall charge for each system of $+3 \mathrm{e}$. Each structure was solvated by placing it in a $30 \AA$ box of 8000 pre-equilibrated TIP3P water molecules. Water molecules at a distance of greater than $25 \AA$ from the iron and whose oxygen atom was closer than $2.6 \AA$ to any heavy protein atoms were deleted.

A series of energy minimizations was carried out for each of the structures. Firstly, the positions of all the hydrogen atoms were minimized, using 100 steps of steepest descent minimization (SD), followed by 500 steps of conjugate gradient minimization (CG). The system was subsequently solvated and truncated. The added water was energy-minimized with 1000 steps of SD and 500 CG; this was followed by a minimization of all the water molecules using 1000 SD and 1500 adopted basis Newton-Raphson minimization (ABNR). A short MD simulation using Langevin dynamics was then carried out for the water molecules; this comprised a heating stage of $1 \mathrm{ps}$ where the temperature was raised to $300 \mathrm{~K}$ and an equilibration stage of $25 \mathrm{ps}$. The end point of this simulation was energy minimized for the water molecules with 500 steps of SD and 1500 steps of ABNR. Finally, each whole protein-ligand-solvent model was energy minimized using 500 steps SD and 1500 steps ABNR. The minimization was carried out with harmonic restraints placed on protein atoms further than $20 \AA$ from the haem iron. The force constant for these restraints increased in value with distance from the iron.

Each of the four minimized systems was heated to $300 \mathrm{~K}$ over $60 \mathrm{ps}$ and equilibrated for 1000 ps before using three different sets of random initial velocities; this yielded a total of twelve MD simulations. Each simulation had a production phase of approximately $8 \mathrm{~ns}$ and a time step of $0.001 \mathrm{ps}$. The SHAKE algorithm was used to constrain all bonds to hydrogen atoms. Structural snapshots were saved every 0.5 ps.

MM parameters for mexiletine:

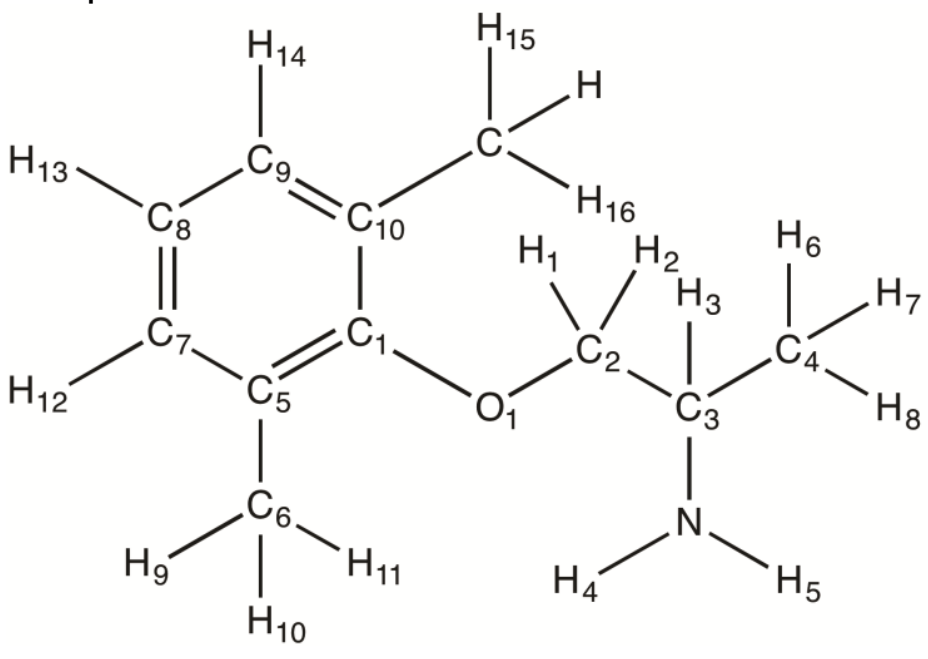

MASS 218 OCE $15.99900 \quad 0$ ! New atom type for ether oxygen

RESI MEX +0.000! type from CHARMM

GROUP 


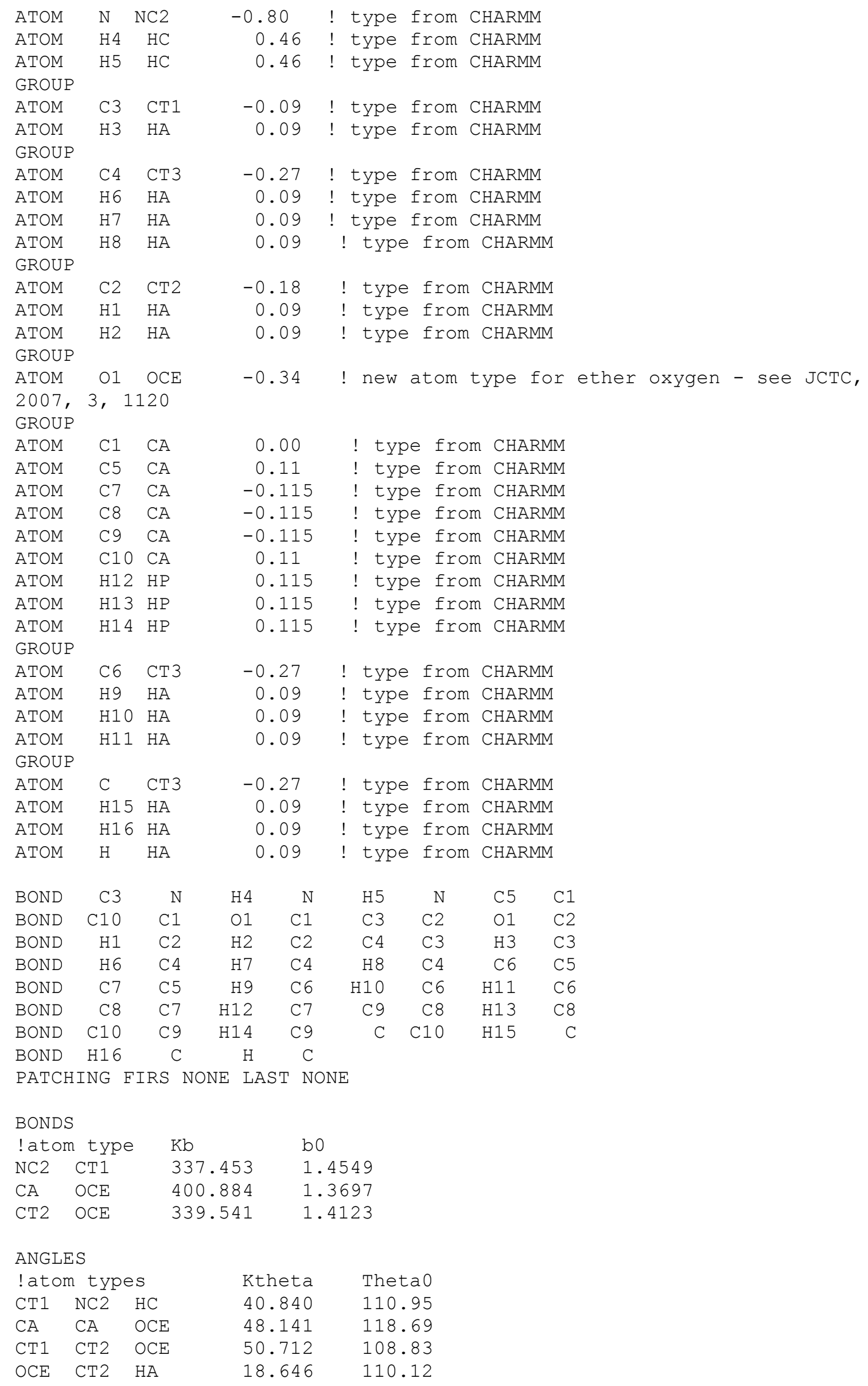




$\begin{array}{lllrr}\mathrm{NC} 2 & \mathrm{CT} 1 & \mathrm{CT} 2 & 20.875 & 113.72 \\ \mathrm{NC} 2 & \mathrm{CT} 1 & \mathrm{CT} 3 & 21.155 & 109.14 \\ \mathrm{NC} 2 & \mathrm{CT} 1 & \mathrm{HA} & 6.837 & 107.13 \\ \mathrm{CA} & \mathrm{OCE} & \mathrm{CT} 2 & 59.807 & 116.49\end{array}$

\section{DIHEDRALS}

! atom types

$\mathrm{HC} \quad \mathrm{NC2} \quad \mathrm{CT} 1 \quad \mathrm{CT} 2$

$\mathrm{HC} \quad \mathrm{NC} 2$ CT1 $\mathrm{CT} 3$

KChi

0.1300

0.1350

0.0950

0.5822

0.5729

1.0661

0.6714

0.8069

0.5445

delta
0.00
0.00
0.00
180.00
0.00
180.00
0.00
0.00
180.00

NONBONDED

$\begin{array}{cccc}\text { !atom } & \text { ignored } & \text { epsilon } & \text { Rmin/2 } \\ \text { OCE } & 0.0 & -0.1521 & 1.7700\end{array}$


Part B: Interatomic distances calculated during molecular dynamics simulations.<smiles>Cc1ccccc1OCC(C)N</smiles>

$$
\begin{aligned}
& 1 \text { - para } \\
& 2 \text { - meta1/meta2 } \\
& \text { 3 - methyl1/methyl2 } \\
& 4 \text { - amine }
\end{aligned}
$$

Figure S1. Labelling of oxidation sites of mexiletine, as used in Figs S2-10.

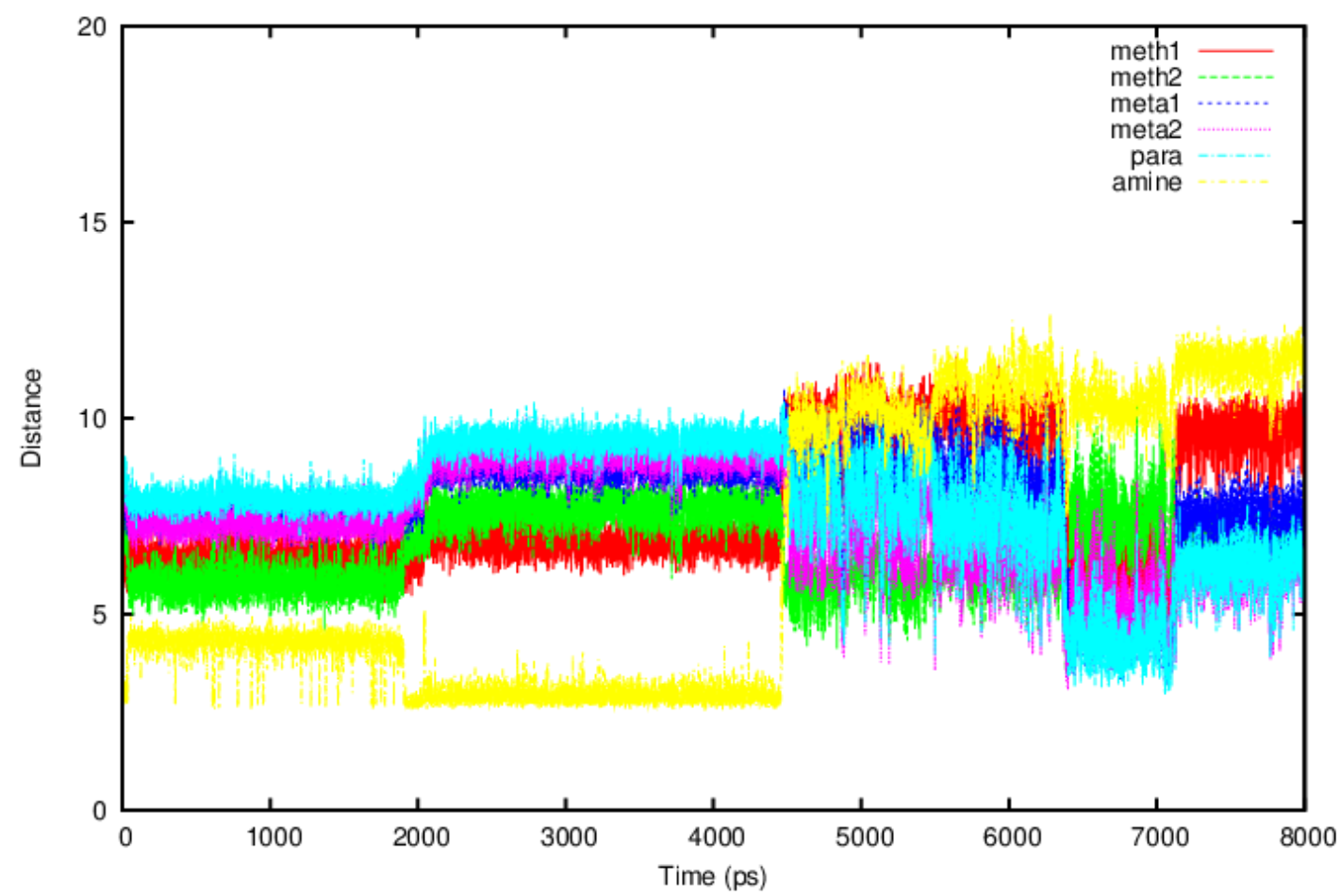

Figure S2: Interatomic distances [in $\AA$ ] between Cpd I oxygen and possible sites of oxidation on mexiletine during molecular dynamics simulations (as depicted in Figure S1). Docking pose A, simulation 1. 


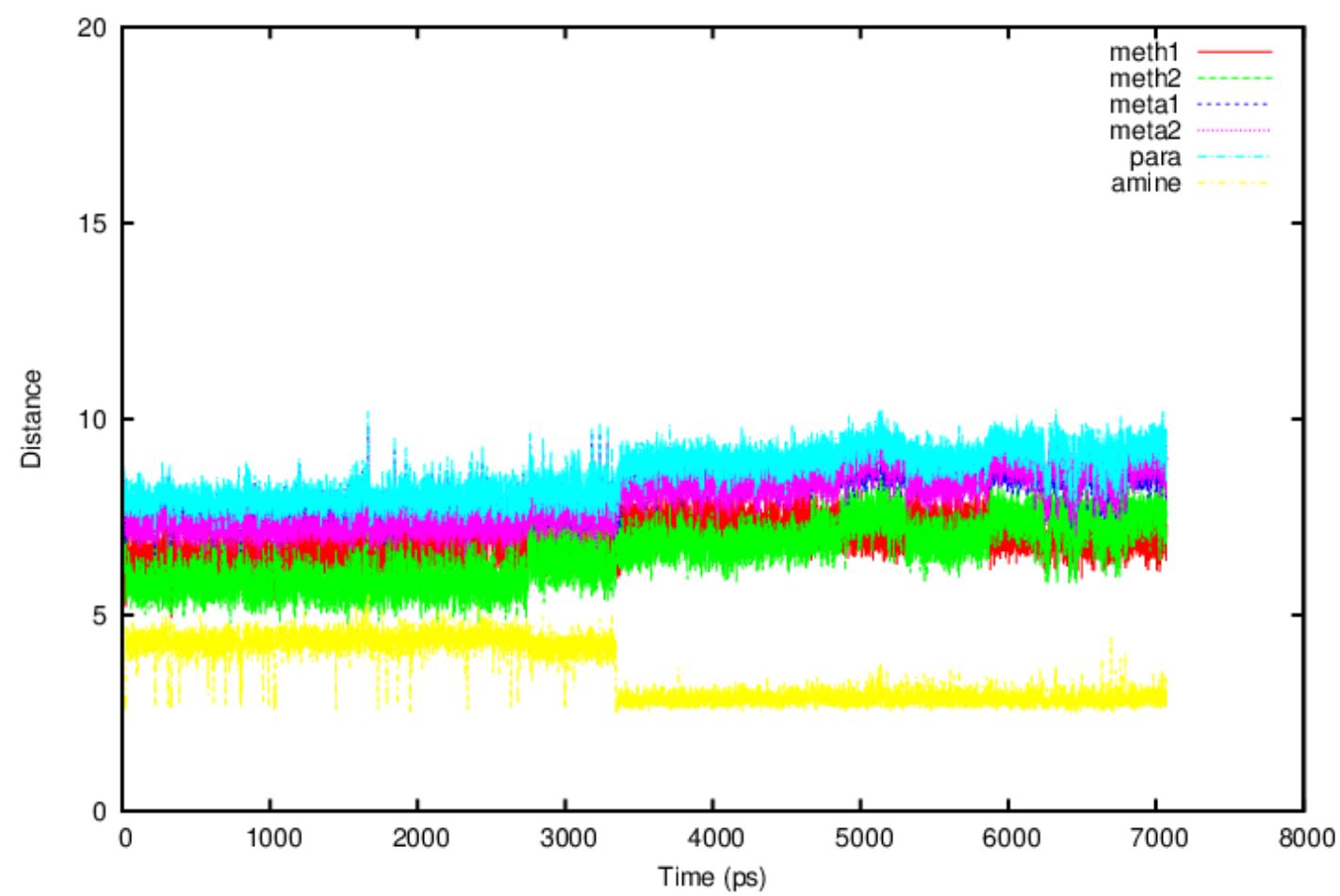

Figure S3: Interatomic distances [in $\AA$ ] between Cpd I oxygen and possible sites of oxidation on mexiletine during molecular dynamics simulations (as depicted in Figure S1). Docking pose A, simulation 2.

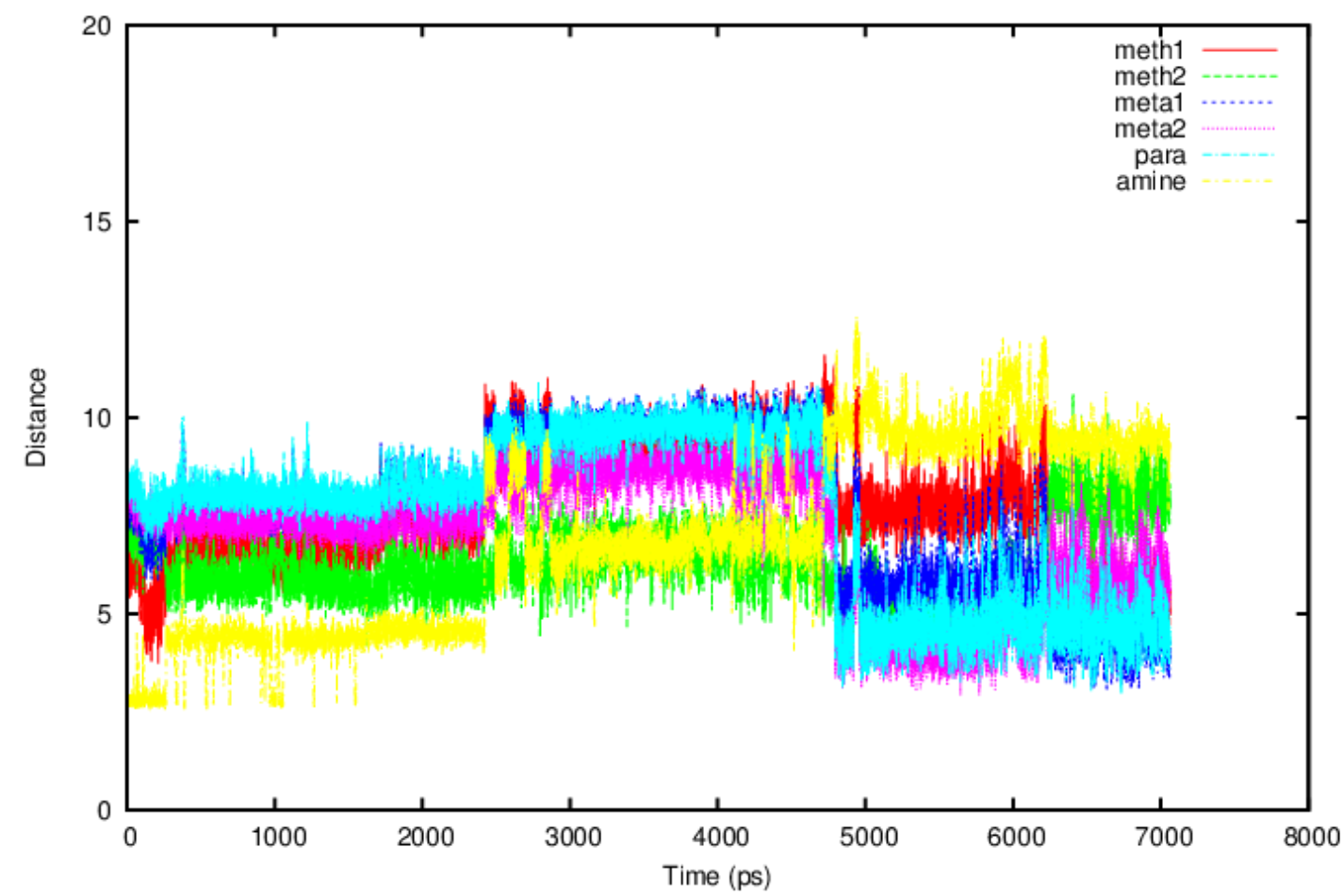

Figure S4: Interatomic distances [in Å] between Cpd I oxygen and possible sites of oxidation on mexiletine during molecular dynamics simulations (as depicted in Figure S1). Docking pose A, simulation 3. 


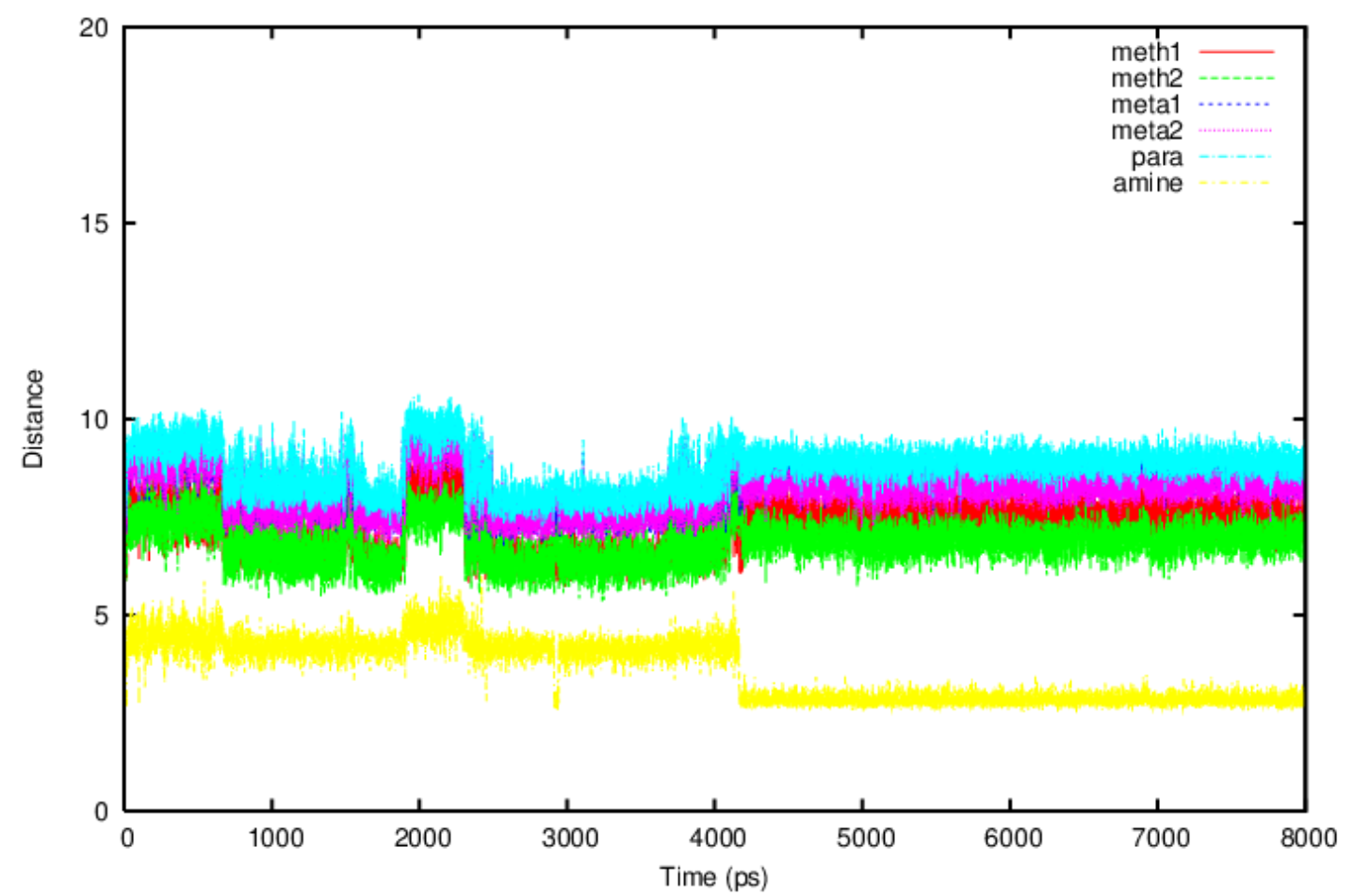

Figure S5: Interatomic distances [in $\AA$ ] between Cpd I oxygen and possible sites of oxidation on mexiletine during molecular dynamics simulations (as depicted in Figure S1). Docking pose B, simulation 1.

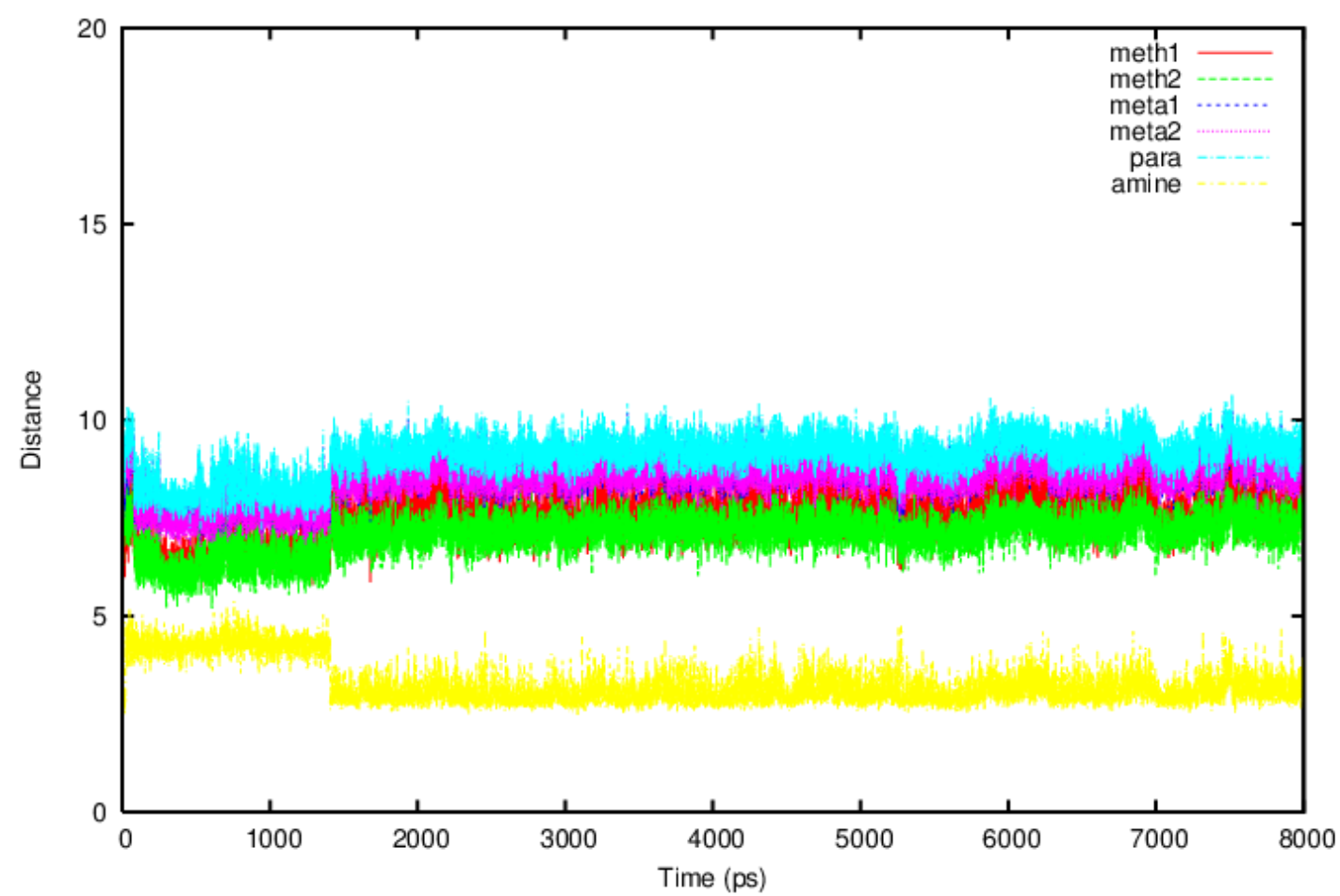

Figure S6: Interatomic distances [in Å] between Cpd I oxygen and possible sites of oxidation on mexiletine during molecular dynamics simulations (as depicted in Figure S1). Docking pose B, simulation 2. 


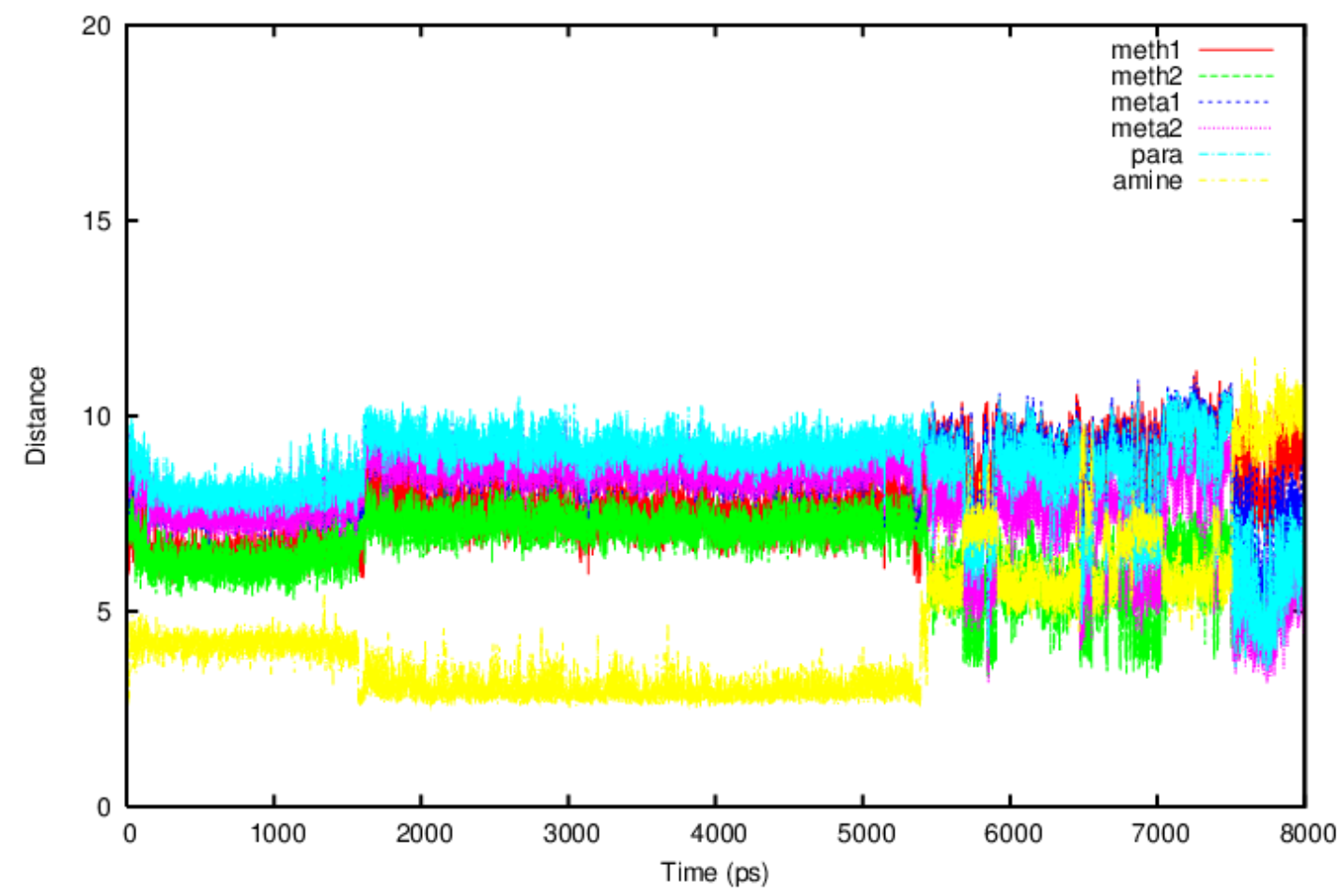

Figure S7: Interatomic distances [in $\AA$ ] between Cpd I oxygen and possible sites of oxidation on mexiletine during molecular dynamics simulations (as depicted in Figure S1). Docking pose B, simulation 3.

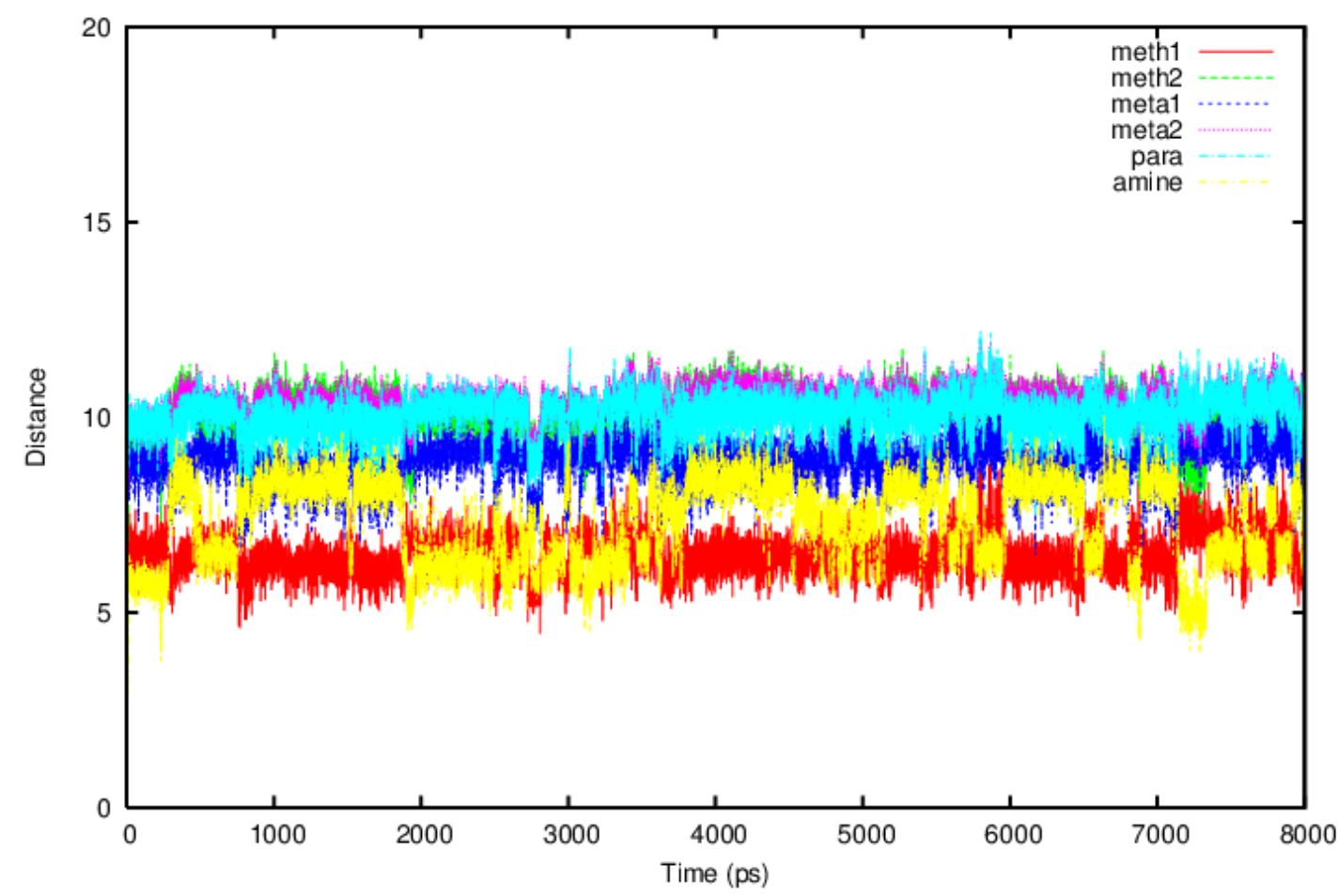

Figure S8: Interatomic distances [in Å] between Cpd I oxygen and possible sites of oxidation on mexiletine during molecular dynamics simulations (as depicted in Figure S1). Docking pose $\mathrm{C}$, simulation 1. 


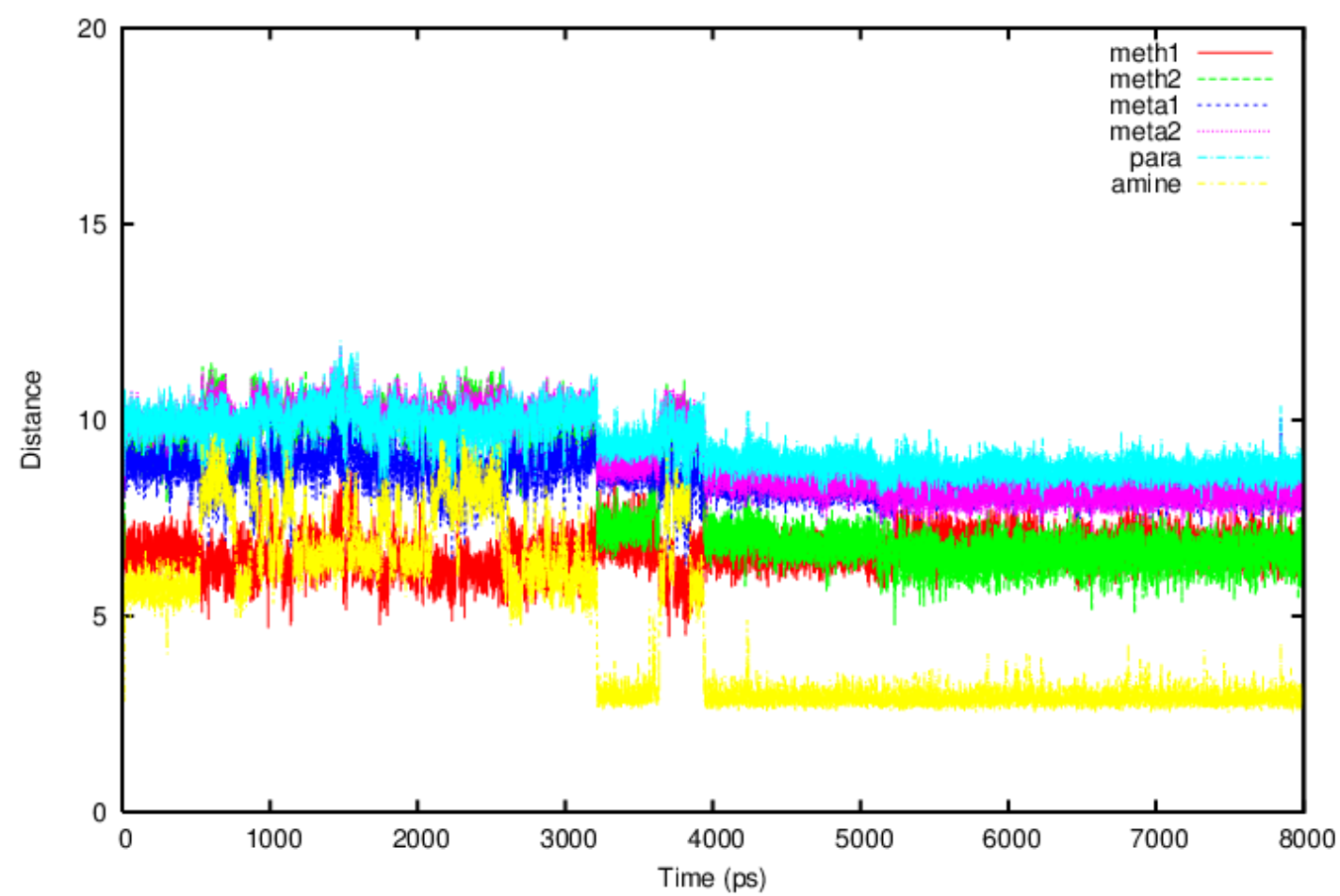

Figure S9: Interatomic distances [in $\AA$ ] between Cpd I oxygen and possible sites of oxidation on mexiletine during molecular dynamics simulations (as depicted in Figure S1). Docking pose $\mathrm{C}$, simulation 2.

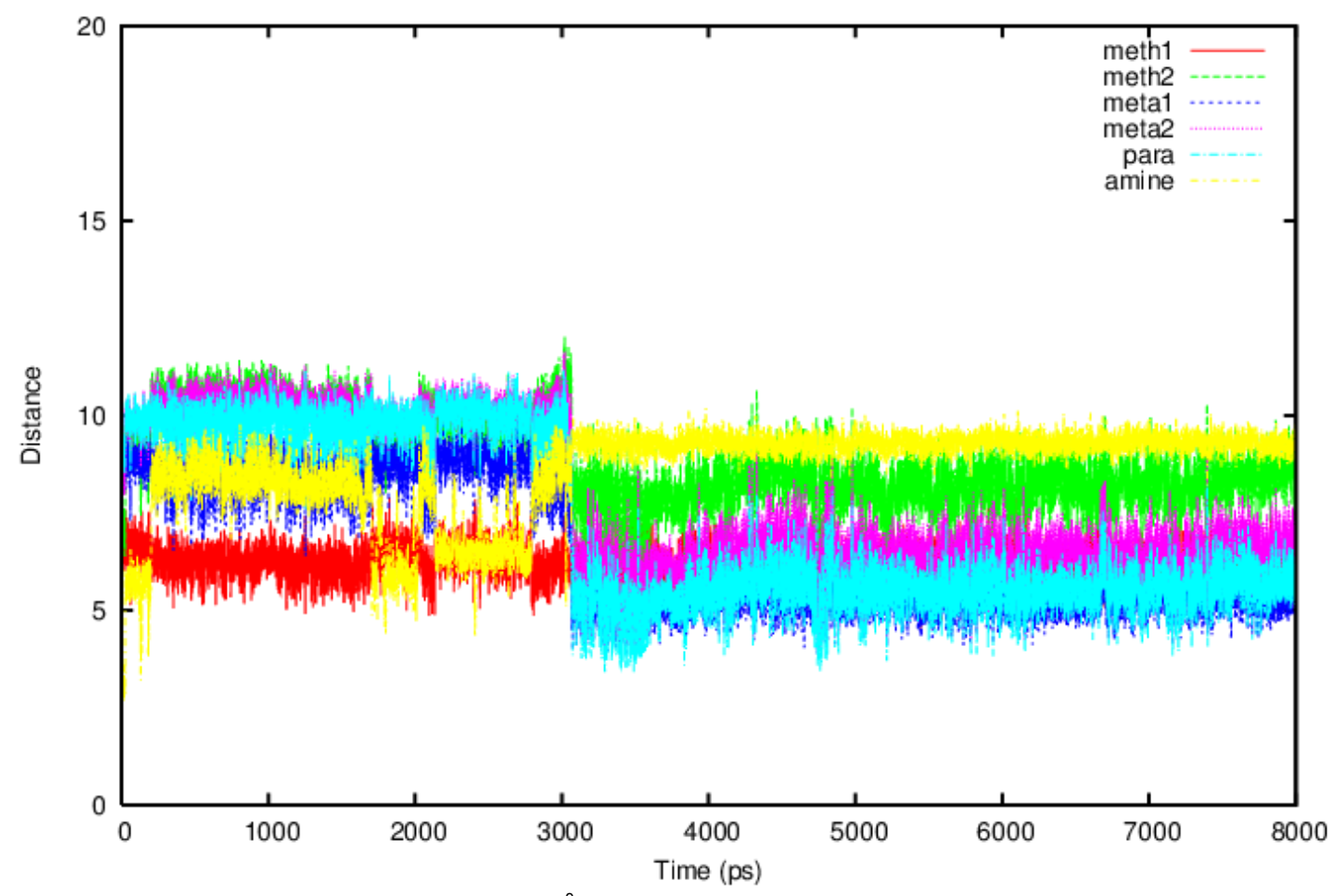

Figure S10: Interatomic distances [in $\AA$ ] between Cpd I oxygen and possible sites of oxidation on mexiletine during molecular dynamics simulations (as depicted in Figure S1). Docking pose $\mathrm{C}$, simulation 3. 


\section{Part C: QM/MM energies of all species (calculated using BSI).}

Table S1. QM/MM energies [in Hartrees] of stationary points involved in $\mathrm{H}$ abstraction from mexiletine (calculated using BSI).

\begin{tabular}{|c|c|c|c|c|}
\hline \multirow{2}{*}{ Pathway } & \multicolumn{2}{|c|}{$\mathrm{RC}_{\text {HABS }}$} & \multicolumn{2}{c|}{ qS $_{\text {HABS }}$} \\
\cline { 2 - 5 } & doublet & quartet & doublet & quartet \\
\hline A1 & -2196.500469 & -2196.499743 & -2196.472535 & -2196.470741 \\
\hline A2 & -2196.696235 & -2196.689729 & -2196.672789 & -2196.665417 \\
\hline A3 & -2196.654068 & -2196.646387 & -2196.624189 & -2196.616513 \\
\hline B1 & -2196.888533 & -2196.881981 & -2196.866016 & -2196.857816 \\
\hline B2 & -2196.734683 & -2196.728678 & -2196.716278 & -2196.710199 \\
\hline B3 & -2196.673753 & -2196.667236 & -2196.654008 & -2196.642958 \\
\hline B4 & -2196.695546 & -2196.700036 & -2196.677670 & -2196.673033 \\
\hline B5 & -2196.602226 & -2196.596060 & -2196.580643 & -2196.575008 \\
\hline C1 & -2196.626941 & -2196.620808 & -2196.595747 & -2196.592168 \\
\hline
\end{tabular}

Table S2. QM/MM energies [in Hartrees] of stationary points involved in the rebound pathway (calculated using BSI).

\begin{tabular}{|c|c|c|c|c|}
\hline \multirow{2}{*}{ Pathway } & \multicolumn{2}{|c|}{ I $_{\text {HABS }}$} & \multicolumn{2}{c|}{ TS REB } \\
\cline { 2 - 5 } & doublet & quartet & doublet & quartet \\
\hline A1 & -2196.485220 & -2196.479003 & -2196.469244 & -2196.463396 \\
\hline A2 & -2196.679212 & -2196.675293 & -2196.665638 & -2196.654531 \\
\hline A3 & -2196.632008 & -2196.626635 & -2196.616156 & -2196.591082 \\
\hline B1 & -2196.882926 & -2196.868499 & -2196.861134 & -2196.838758 \\
\hline B2 & -2196.723731 & -2196.717240 & -2196.702316 & -2196.689551 \\
\hline B3 & -2196.659865 & -2196.650509 & -2196.644400 & -2196.620533 \\
\hline B4 & -2196.690799 & -2196.684982 & -2196.668870 & -2196.653443 \\
\hline B5 & -2196.589697 & -2196.583763 & -2196.560224 & -2196.547754 \\
\hline C1 & -2196.607514 & -2196.602409 & -2196.595887 & -2196.588127 \\
\hline
\end{tabular}

Table S3. QM/MM energies [in Hartrees] of $\mathrm{H}$-abstraction/rebound product (calculated using BSI).

\begin{tabular}{|c|c|c|}
\hline \multirow{2}{*}{ Pathway } & doublet & PREB \\
\cline { 2 - 3 } & -2196.534284 & -2196.511589 \\
\hline A1 & -2196.737300 & -2196.710423 \\
\hline A2 & -2196.680065 & -2196.655453 \\
\hline A3 & -2196.925971 & -2196.914100 \\
\hline B1 & -2196.785335 & -2196.761713 \\
\hline B2 & -2196.721813 & -2196.698174 \\
\hline B3 & -2196.739965 & -2196.729719 \\
\hline B4 & -2196.634020 & -2196.626129 \\
\hline B5 & -2196.657335 & -2196.648376 \\
\hline C1 & \multicolumn{2}{|}{} \\
\hline
\end{tabular}


Table S4. QM/MM energies [in Hartrees] of stationary points involved in $\mathrm{N}$-oxidation of mexiletine (calculated using BSI).

\begin{tabular}{|c|c|c|c|c|}
\hline \multirow{2}{*}{ Pathway } & \multicolumn{2}{|c|}{$\mathrm{RC}_{\text {NOX }}$} & \multicolumn{2}{c|}{ qS $_{\text {NOX }}$} \\
\cline { 2 - 5 } & doublet & quartet & doublet & quartet \\
\hline A1 & -2196.500469 & -2196.499743 & -2196.484259 & -2196.463563 \\
\hline A2 & -2196.696236 & -2196.689849 & -2196.681207 & -2196.657260 \\
\hline A3 & -2196.654068 & -2196.646387 & -2196.631352 & -2196.601525 \\
\hline B1 & -2196.888533 & -2196.881002 & -2196.872612 & -2196.857045 \\
\hline B2 & -2196.734683 & -2196.728678 & -2196.706247 & -2196.698860 \\
\hline B3 & -2196.673753 & -2196.667236 & -2196.637791 & -2196.620788 \\
\hline B4 & -2196.688547 & -2196.697040 & -2196.670004 & -2196.654989 \\
\hline B5 & -2196.602226 & -2196.594338 & -2196.572563 & -2196.560261 \\
\hline C1 & -2196.626941 & -2196.620808 & -2196.593336 & -2196.570355 \\
\hline
\end{tabular}

Table S5. QM/MM energies [in Hartrees] of $\mathrm{N}$-oxidation product (calculated using $\mathrm{BSI})$.

\begin{tabular}{|c|c|c|}
\hline \multirow{2}{*}{ Pathway } & \multicolumn{2}{|c|}{$\mathrm{P}_{\text {NOX }}$} \\
\cline { 2 - 3 } & doublet & quartet \\
\hline A1 & -2196.534779 & -2196.511900 \\
\hline A2 & -2196.736566 & -2196.708695 \\
\hline A3 & -2196.679862 & -2196.655816 \\
\hline B1 & -2196.931095 & -2196.893047 \\
\hline B2 & -2196.771326 & -2196.750039 \\
\hline B3 & -2196.702339 & -2196.678670 \\
\hline B4 & -2196.729756 & -2196.705166 \\
\hline B5 & -2196.632792 & -2196.608654 \\
\hline C1 & -2196.647433 & -2196.613743 \\
\hline
\end{tabular}




\section{Part D: QM/MM energies of all species (calculated using BSII).}

Table S6. QM/MM energies [in Hartrees] of stationary points involved in $\mathrm{H}$ abstraction from mexiletine (calculated using BSII//BSI).

\begin{tabular}{|c|c|c|c|c|}
\hline \multirow{2}{*}{ Pathway } & \multicolumn{2}{|c|}{ RC $_{\text {HABS }}$} & \multicolumn{2}{c|}{ TS $_{\text {HABS }}$} \\
\cline { 2 - 5 } & doublet & quartet & doublet & quartet \\
\hline A1 & -2197.011842 & -2197.015113 & -2196.987894 & -2196.990552 \\
\hline A2 & -2197.214523 & -2197.207329 & -2197.191576 & -2197.183584 \\
\hline A3 & -2197.171028 & -2197.162813 & -2197.142725 & -2197.134602 \\
\hline B1 & -2197.408994 & -2197.401685 & -2197.386317 & -2197.377824 \\
\hline B2 & -2197.252840 & -2197.246263 & -2197.241839 & -2197.234844 \\
\hline B3 & -2197.192023 & -2197.184769 & -2197.178263 & -2197.160992 \\
\hline B4 & -2197.207263 & -2197.215661 & -2197.196989 & -2197.192231 \\
\hline B5 & -2197.120624 & -2197.114049 & -2197.107392 & -2197.100804 \\
\hline C1 & -2197.143966 & -2197.137286 & -2197.118628 & -2197.113983 \\
\hline
\end{tabular}

Table S7. QM/MM energies [in Hartrees] of stationary points involved in the rebound pathway (calculated using BSII/BSI).

\begin{tabular}{|c|c|c|c|c|}
\hline \multirow{2}{*}{ Pathway } & \multicolumn{2}{|c|}{$\mathrm{I}_{\text {REB }}$} & \multicolumn{2}{c|}{ TS } \\
\cline { 2 - 5 } REB & doublet & quartet & doublet & quartet \\
\hline A1 & -2197.010293 & -2197.003350 & -2196.992890 & -2196.985828 \\
\hline A2 & -2197.201695 & -2197.195259 & -2197.185085 & -2197.173363 \\
\hline A3 & -2197.151288 & -2197.145682 & -2197.134721 & -2197.108891 \\
\hline B1 & -2197.396180 & -2197.389822 & -2197.382157 & -2197.359736 \\
\hline B2 & -2197.250223 & -2197.243114 & -2197.22739 & -2197.214192 \\
\hline B3 & -2197.185870 & -2197.170029 & -2197.169099 & -2197.140919 \\
\hline B4 & -2197.211439 & -2197.205670 & -2197.188722 & -2197.174223 \\
\hline B5 & -2197.116977 & -2197.110518 & -2197.085997 & -2197.073360 \\
\hline C1 & -2197.133320 & -2197.127576 & -2197.120972 & -2197.113531 \\
\hline
\end{tabular}

Table S8. QM/MM energies [in Hartrees] of $\mathrm{H}$-abstraction/rebound product (calculated using BSII/BSI).

\begin{tabular}{|c|c|c|}
\hline \multirow{2}{*}{ Pathway } & \multicolumn{2}{|c|}{ PHABS } \\
\cline { 2 - 3 } & doublet & quartet \\
\hline A1 & -2197.051977 & -2197.032270 \\
\hline A2 & -2197.256233 & -2197.232347 \\
\hline A3 & -2197.197950 & -2197.176055 \\
\hline B1 & -2197.446031 & -2197.436698 \\
\hline B2 & -2197.304084 & -2197.283703 \\
\hline B3 & -2197.240538 & -2197.220239 \\
\hline B4 & -2197.259807 & $-2,197.251144$ \\
\hline B5 & -2197.153850 & -2197.148578 \\
\hline C1 & -2197.177697 & -2197.171355 \\
\hline
\end{tabular}


Table S9. QM/MM energies [in Hartrees] of stationary points involved in $\mathrm{N}$-oxidation of mexiletine (calculated using BSII/BSI).

\begin{tabular}{|c|c|c|c|c|}
\hline \multirow{2}{*}{ Pathway } & \multicolumn{2}{|c|}{$\mathrm{RC}_{\text {NOX }}$} & \multicolumn{2}{c|}{ TS $_{\text {NOX }}$} \\
\cline { 2 - 5 } & doublet & quartet & doublet & quartet \\
\hline A1 & -2197.015632 & -2197.015113 & -2197.003308 & -2196.980638 \\
\hline A2 & -2197.214523 & -2197.207329 & -2197.196778 & -2197.170604 \\
\hline A3 & -2197.171028 & -2197.162813 & -2197.153544 & -2197.114461 \\
\hline B1 & -2197.408994 & -2197.401685 & -2197.389967 & -2197.371735 \\
\hline B2 & -2197.25284 & -2197.246263 & -2197.221725 & -2197.218348 \\
\hline B3 & -2197.192023 & -2197.184769 & -2197.153810 & -2197.135655 \\
\hline B4 & -2197.207263 & -2197.215661 & -2197.186905 & -2197.170347 \\
\hline B5 & -2197.120624 & -2197.114049 & -2197.088374 & -2197.079827 \\
\hline C1 & -2197.143966 & -2197.137286 & -2197.107759 & -2197.084010 \\
\hline
\end{tabular}

Table S10. QM/MM energies [in Hartrees] of $\mathrm{N}$-oxidation product (calculated using $\mathrm{BSII} / \mathrm{BSI})$.

\begin{tabular}{|c|c|c|}
\hline \multirow{2}{*}{ Pathway } & \multicolumn{2}{|c|}{$\mathrm{P}_{\text {NOX }}$} \\
\cline { 2 - 3 } & doublet & quartet \\
\hline A1 & -2197.052460 & -2197.032726 \\
\hline A2 & -2197.255540 & -2197.231041 \\
\hline A3 & -2197.197731 & -2196.975638 \\
\hline B1 & -2197.450576 & -2197.407140 \\
\hline B2 & -2197.291241 & -2197.273677 \\
\hline B3 & -2197.221811 & -2197.202226 \\
\hline B4 & -2197.251404 & -2197.229223 \\
\hline B5 & -2197.153177 & -2197.133225 \\
\hline C1 & -2197.166685 & -2197.136436 \\
\hline
\end{tabular}




\section{Part E: QM/MM energies calculated with the BHandHLYP and BP86 density functionals}

Table S11. Single point QM/MM energies [in Hartrees] calculated on stationary points for the doublet $\mathrm{H}$-abstraction pathway $\mathrm{B}-2$ with different functionals. (BSI)

\begin{tabular}{|c|c|c|c|c|c|}
\hline Functional & $\mathrm{RC}$ & $\mathrm{TS}_{\text {HAB }}$ & $\mathrm{I}_{\text {HABS }}$ & $\mathrm{TS}_{\text {REB }}$ & $\mathrm{P}_{\text {HABS }}$ \\
\hline B3LYP-D & -2196.734683 & -2196.716278 & -2196.723731 & -2196.702316 & -2196.78534 \\
\hline B3LYP & -2196.624203 & -2196.602741 & -2196.610446 & -2196.585905 & -2196.663214 \\
\hline BP86 & -2196.765191 & -2196.75286 & -2196.752001 & -2196.735829 & -2196.791377 \\
\hline BHandHLYP & -2177.556925 & -2177.549399 & -2177.563188 & -2177.538456 & -2177.634987 \\
\hline
\end{tabular}

Table S12. Single point QM/MM energies [in Hartrees] calculated on stationary points for the quartet $\mathrm{H}$-abstraction pathway $\mathrm{B}-2$ with different functionals. (BSI)

\begin{tabular}{|c|c|c|c|c|c|}
\hline Functional & RC & TS $_{\text {HAB }}$ & I $_{\text {HABS }}$ & TS $_{\text {REB }}$ & P $_{\text {HABS }}$ \\
\hline B3LYP-D & -2196.728678 & -2196.710199 & -2196.75026 & -2196.689551 & -2196.761873 \\
\hline B3LYP & -2196.618198 & -2196.586389 & -2196.637752 & -2196.596234 & -2196.603612 \\
\hline BP86 & -2196.762521 & -2196.737534 & -2196.753344 & -2196.749844 & -2196.747932 \\
\hline BHandHLYP & -2177.543423 & -2177.520482 & -2177.623169 & -2177.532577 & -2177.546577 \\
\hline
\end{tabular}

Table S13. Single point QM/MM energies [in Hartrees] calculated on stationary points for the doublet $\mathrm{N}$-oxidation pathway B-2 with different functionals. (BSI)

\begin{tabular}{|c|c|c|}
\hline Functional & TS $_{\text {NOX }}$ & P $_{\text {NOX }}$ \\
\hline B3LYP-D & -2196.706247 & -2196.771326 \\
\hline B3LYP & -2196.596147 & -2196.65362 \\
\hline BP86 & -2196.746712 & -2196.780432 \\
\hline BHandHLYP & -2177.536321 & -2177.62314 \\
\hline
\end{tabular}

Table S14. Single point QM/MM energies [in Hartrees] calculated on stationary points for the quartet $\mathrm{N}$-oxidation pathway B-2 with different functionals. (BSI)

\begin{tabular}{|c|c|c|}
\hline Functional & TS $_{\text {NOX }}$ & P $_{\text {NOX }}$ \\
\hline B3LYP-D & -2196.698860 & -2196.750039 \\
\hline B3LYP & -2196.586389 & -2196.637752 \\
\hline BP86 & -2196.737534 & -2196.753344 \\
\hline BHandHLYP & -2177.520482 & -2177.623169 \\
\hline
\end{tabular}

Table S15. QM/MM energies [in $\mathrm{kcal} / \mathrm{mol}$ ] of stationary points for the doublet $\mathrm{H}$ abstraction pathway B-2 with different functionals, relative to reactant complex, RC. (BSI)

\begin{tabular}{|c|c|c|c|c|c|}
\hline Functional & $\mathrm{RC}$ & $\mathrm{TS}_{\text {HAB }}$ & $\mathrm{I}_{\text {HABS }}$ & $\mathrm{TS}_{\text {REB }}$ & $\mathrm{P}_{\text {HABS }}$ \\
\hline B3LYP-D & 0 & 11.55 & 6.87 & 20.31 & -31.79 \\
\hline B3LYP & 0 & 13.47 & 8.63 & 24.03 & -24.48 \\
\hline BP86 & 0 & 7.74 & 8.28 & 18.42 & -16.43 \\
\hline BHandHLYP & 0 & 4.72 & -3.93 & 11.59 & -48.98 \\
\hline
\end{tabular}

Table S16. QM/MM energies [in $\mathrm{kcal} / \mathrm{mol}$ ] of stationary points for the quartet $\mathrm{H}$ abstraction pathway B-2 with different functionals, relative to reactant complex, RC. (BSI)

\begin{tabular}{|c|c|c|c|c|c|}
\hline Functional & $\mathrm{RC}$ & $\mathrm{TS}_{\text {HAB }}$ & $\mathrm{I}_{\text {HABS }}$ & TS $_{\text {REB }}$ & $\mathrm{P}_{\text {HABS }}$ \\
\hline B3LYP-D & 0 & 11.60 & 7.17 & 24.55 & -20.83 \\
\hline B3LYP & 0 & 13.78 & 9.15 & 27.40 & -15.60 \\
\hline BP86 & 0 & 7.95 & 9.15 & 23.10 & 2.06 \\
\hline BHandHLYP & 0 & 6.81 & -1.98 & 19.69 & -56.05 \\
\hline
\end{tabular}


Table S17. QM/MM energies [in $\mathrm{kcal} / \mathrm{mol}$ ] of stationary points for the doublet $\mathrm{N}$ oxdiation pathway B-2 with different functionals, relative to reactant complex, $\mathrm{RC}$. (BSI)

\begin{tabular}{|c|c|c|}
\hline Functional & TS $_{\text {NOX }}$ & P $_{\text {NOX }}$ \\
\hline B3LYP-D & 17.84 & -22.99 \\
\hline B3LYP & 17.61 & -18.46 \\
\hline BP86 & 11.60 & -9.56 \\
\hline BHandHLYP & 12.93 & -41.55 \\
\hline
\end{tabular}

Table S18. QM/MM energies [in $\mathrm{kcal} / \mathrm{mol}$ ] of stationary points for the quartet $\mathrm{N}$ oxdiation pathway B-2 with different functionals, relative to reactant complex, $\mathrm{RC}$. (BSI)

\begin{tabular}{|c|c|c|}
\hline Functional & TS $_{\text {NOX }}$ & P $_{\text {NOX }}$ \\
\hline B3LYP-D & 18.71 & -13.40 \\
\hline B3LYP & 19.96 & -12.27 \\
\hline BP86 & 15.68 & 5.76 \\
\hline BHandHLYP & 14.40 & -50.04 \\
\hline
\end{tabular}

Table S19. Single point QM/MM energies [in Hartrees] calculated on stationary points for the doublet $\mathrm{H}$-abstraction pathway $\mathrm{B}-3$ with different functionals. (BSI)

\begin{tabular}{|c|c|c|c|c|c|}
\hline Functional & $\mathrm{RC}$ & $\mathrm{TS}_{\text {HAB }}$ & $\mathrm{I}_{\text {HABS }}$ & $\mathrm{TS}_{\text {REB }}$ & $\mathrm{P}_{\text {HABS }}$ \\
\hline B3LYP-D & -2196.673753 & -2196.654008 & -2196.659865 & -2196.644400 & -2196.721813 \\
\hline B3LYP & -2196.560726 & -2196.525983 & -2196.582142 & -2196.537666 & -2196.543922 \\
\hline BP86 & -2196.702543 & -2196.674102 & -2196.710567 & -2196.690423 & -2196.686158 \\
\hline BHandHLYP & -2177.496156 & -2177.471358 & -2177.552835 & -2177.500063 & -2177.498445 \\
\hline
\end{tabular}

Table S20. Single point QM/MM energies [in Hartrees] calculated on stationary points for the quartet $\mathrm{H}$-abstraction pathway $\mathrm{B}-3$ with different functionals. (BSI)

\begin{tabular}{|c|c|c|c|c|c|}
\hline Functional & RC & TS $_{\text {HAB }}$ & I $_{\text {HABS }}$ & TS $_{\text {REB }}$ & P $_{\text {HABS }}$ \\
\hline B3LYP-D & -2196.667236 & -2196.642958 & -2196.650509 & -2196.620533 & -2196.698174 \\
\hline B3LYP & -2196.55423 & -2196.508501 & -2196.56452 & -2196.526058 & -2196.534433 \\
\hline BP86 & -2196.696812 & -2196.655357 & -2196.681215 & -2196.677435 & -2196.671511 \\
\hline BHandHLYP & -2177.491054 & -2177.450703 & -2177.549656 & -2177.467155 & -2177.490876 \\
\hline
\end{tabular}

Table S21. Single point QM/MM energies [in Hartrees] calculated on stationary points for the doublet N-oxidation pathway B-3 with different functionals. (BSI)

\begin{tabular}{|c|c|c|}
\hline Functional & TS $_{\text {NOX }}$ & $P_{\text {NOX }}$ \\
\hline B3LYP-D & -2196.637791 & -2196.702339 \\
\hline B3LYP & -2196.526651 & -2196.582861 \\
\hline BP86 & -2196.674102 & -2196.710567 \\
\hline BHandHLYP & -2177.471358 & -2177.552835 \\
\hline
\end{tabular}

Table S22. Single point QM/MM energies [in Hartrees] calculated on stationary points for the quartet N-oxidation pathway B-3 with different functionals. (BSI)

\begin{tabular}{|c|c|c|}
\hline Functional & TS $_{\text {NOX }}$ & $\mathrm{P}_{\text {NOX }}$ \\
\hline B3LYP-D & -2196.620788 & -2196.678670 \\
\hline B3LYP & -2196.509228 & -2196.564787 \\
\hline BP86 & -2196.655357 & -2196.681215 \\
\hline BHandHLYP & -2177.450703 & -2177.549656 \\
\hline
\end{tabular}


Table S23. QM/MM energies [in $\mathrm{kcal} / \mathrm{mol}$ ] of stationary points for the doublet $\mathrm{H}$ abstraction pathway B-3 with different functionals, relative to reactant complex, RC. (BSI)

\begin{tabular}{|c|c|c|c|c|c|}
\hline Functional & $\mathrm{RC}$ & $\mathrm{TS}_{\text {HAB }}$ & $\mathrm{I}_{\text {HABS }}$ & $\mathrm{TS}_{\text {REB }}$ & $\mathrm{P}_{\text {HABS }}$ \\
\hline B3LYP-D & 0 & 12.39 & 8.71 & 18.42 & -30.16 \\
\hline B3LYP & 0 & 14.47 & 10.54 & 21.02 & -23.56 \\
\hline BP86 & 0 & 7.61 & 10.28 & 16.67 & -15.54 \\
\hline BHandHLYP & 0 & -2.45 & -1.44 & 10.45 & -46.78 \\
\hline
\end{tabular}

Table S24. QM/MM energies [in $\mathrm{kcal} / \mathrm{mol}$ ] of stationary points for the quartet $\mathrm{H}$ abstraction pathway B-3 with different functionals, relative to reactant complex, RC. (BSI)

\begin{tabular}{|c|c|c|c|c|c|}
\hline Functional & $\mathrm{RC}$ & $\mathrm{TS}_{\text {HAB }}$ & $\mathrm{I}_{\text {HABS }}$ & $\mathrm{TS}_{\text {REB }}$ & $\mathrm{P}_{\text {HABS }}$ \\
\hline B3LYP-D & 0 & 15.23 & 10.50 & 29.31 & -19.41 \\
\hline B3LYP & 0 & 17.68 & 12.42 & 31.74 & -18.18 \\
\hline BP86 & 0 & 12.16 & 15.88 & 29.99 & -1.24 \\
\hline BHandHLYP & 0 & 15.00 & 0.11 & 21.55 & -46.21 \\
\hline
\end{tabular}

Table S25. QM/MM energies [in $\mathrm{kcal} / \mathrm{mol}$ ] of stationary points for the doublet $\mathrm{N}$ oxdiation pathway B-3 with different functionals, relative to reactant complex, RC. (BSI)

\begin{tabular}{|c|c|c|}
\hline Functional & TS $_{\text {NOX }}$ & $\mathrm{P}_{\text {NOX }}$ \\
\hline B3LYP-D & 22.57 & -17.94 \\
\hline B3LYP & 21.80 & -13.44 \\
\hline BP86 & 17.85 & -5.04 \\
\hline BHandHLYP & 15.56 & -35.57 \\
\hline
\end{tabular}

Table S26. QM/MM energies [in $\mathrm{kcal} / \mathrm{mol}$ ] of stationary points for the quartet $\mathrm{N}$ oxdiation pathway B-3 with different functionals, relative to reactant complex, RC. (BSI)

\begin{tabular}{|c|c|c|}
\hline Functional & TS $_{\text {NOX }}$ & $\mathrm{P}_{\text {NOX }}$ \\
\hline B3LYP-D & 29.15 & -7.17 \\
\hline B3LYP & 28.70 & -6.46 \\
\hline BP86 & 26.01 & 9.79 \\
\hline BHandHLYP & 25.32 & -36.77 \\
\hline
\end{tabular}


Part F: QM geometries of stationary points for profile B1 (calculated with B3LYP-D-LACVP,6-31G(d)/CHARMM27)

$\mathrm{RC}_{\text {NOX }}$ (quartet)

$\mathrm{RC}_{\text {HABS }}$ (quartet)
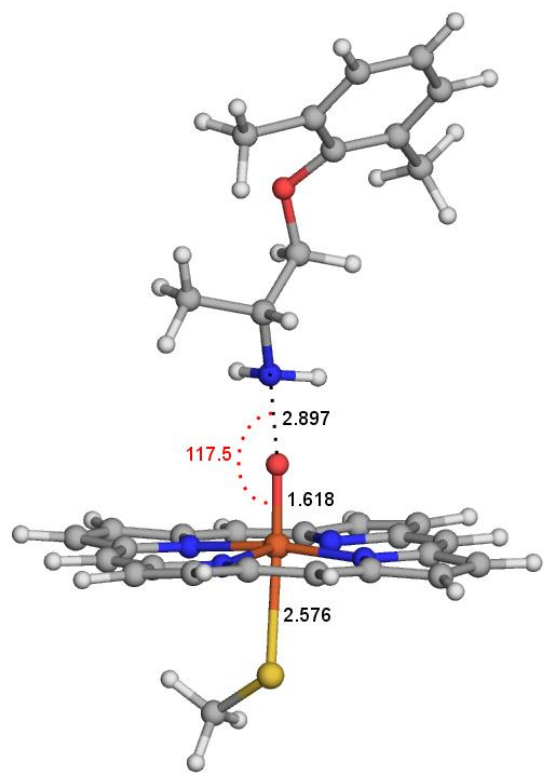

$\mathrm{RC}_{\text {HABS }}$ (doublet)

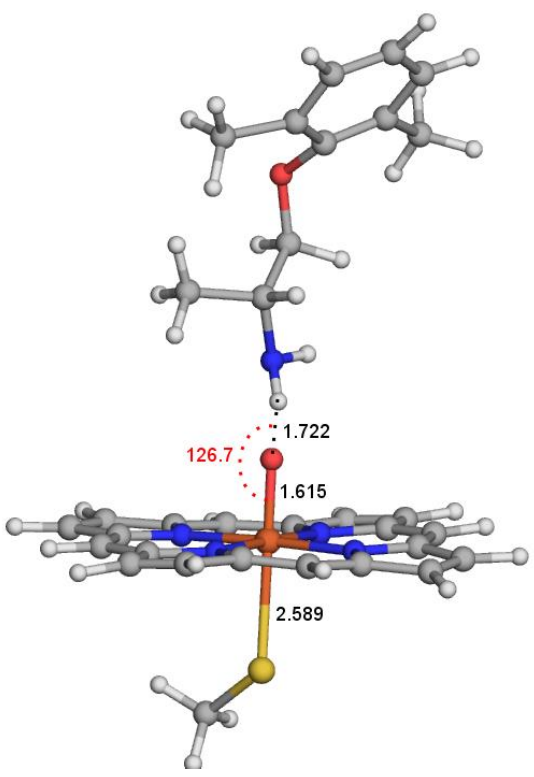

TS $_{\text {HABS }}$ (quartet)
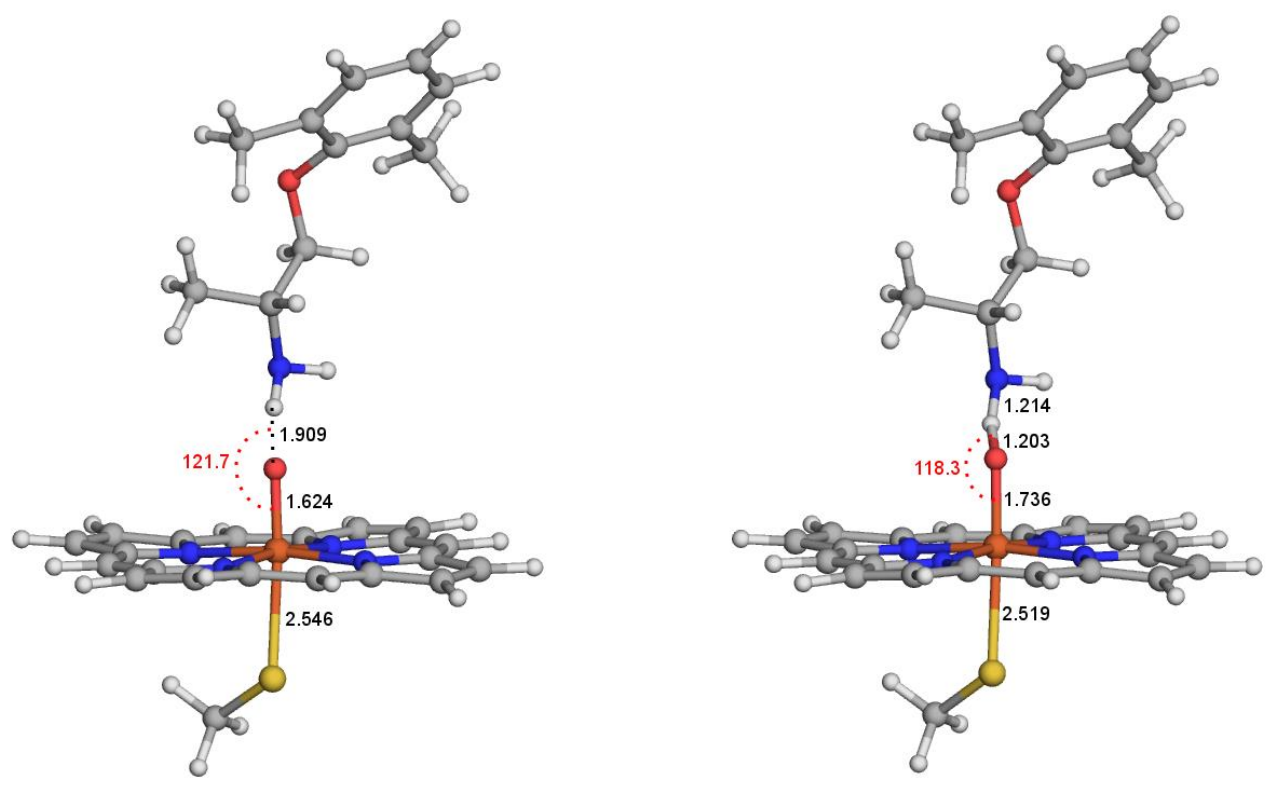
TS $_{\text {HABS }}$ (doublet)

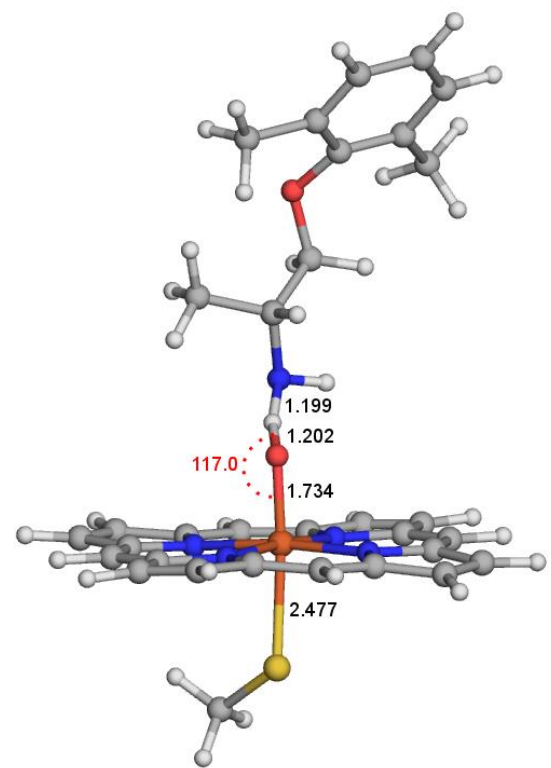

$\mathrm{TS}_{\text {NOx }}$ (doublet)
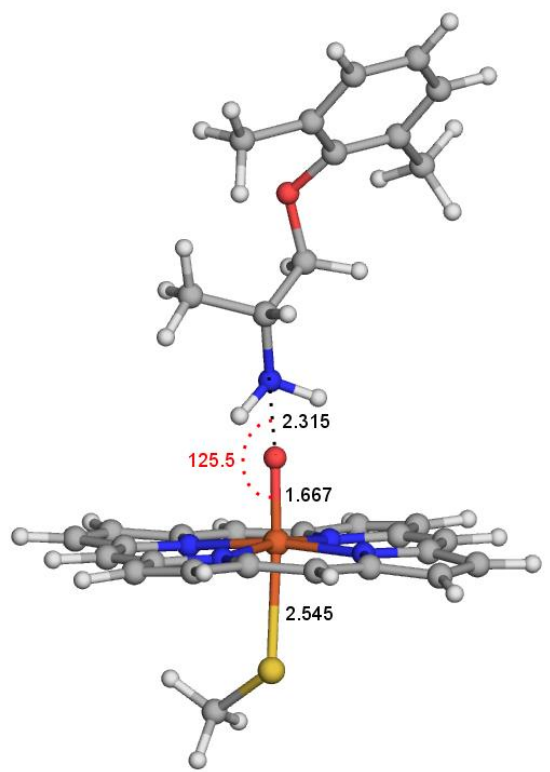

$\mathrm{TS}_{\text {NOx }}$ (quartet)

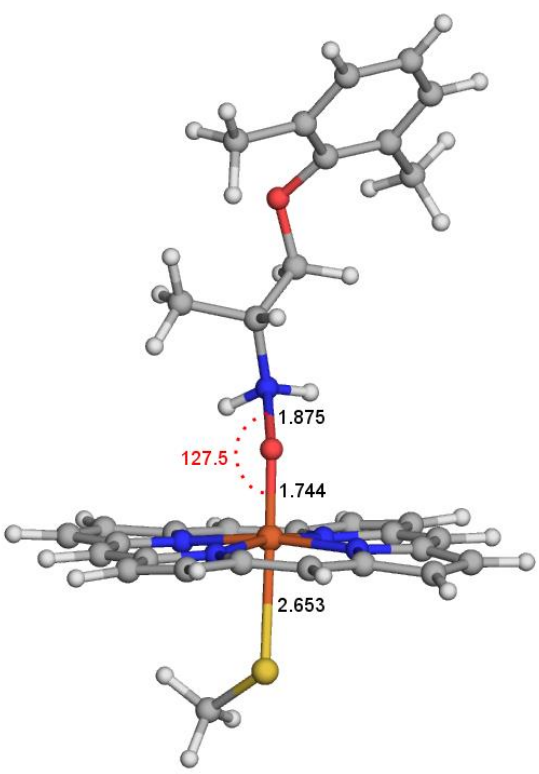

I HABS (quartet)

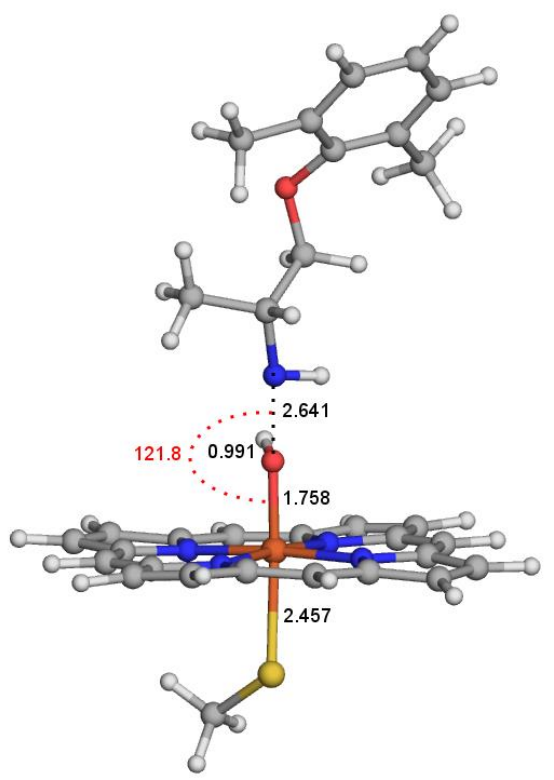


I HABS (doublet)

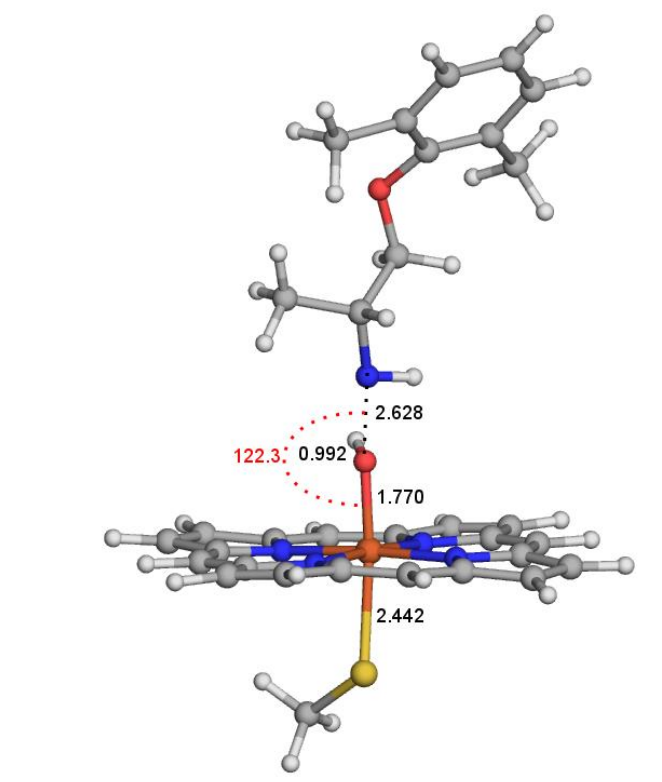

INOX (doublet)

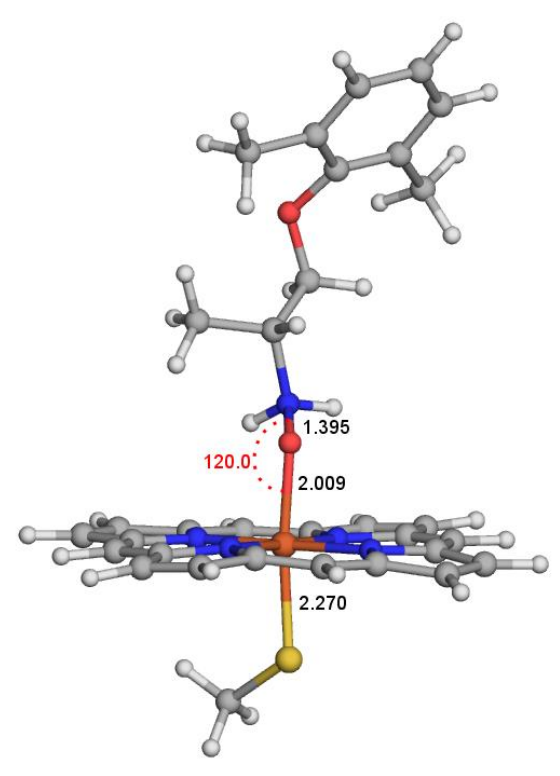

INOX (quartet)

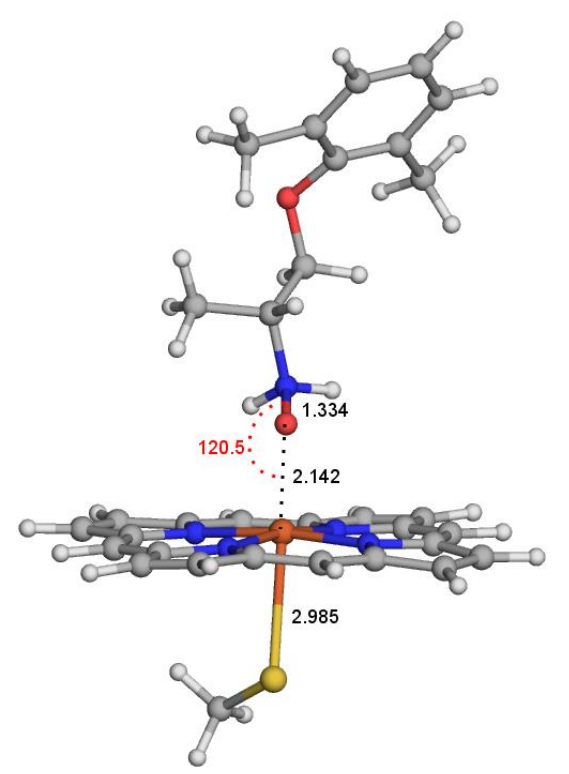

TS REB (quartet)

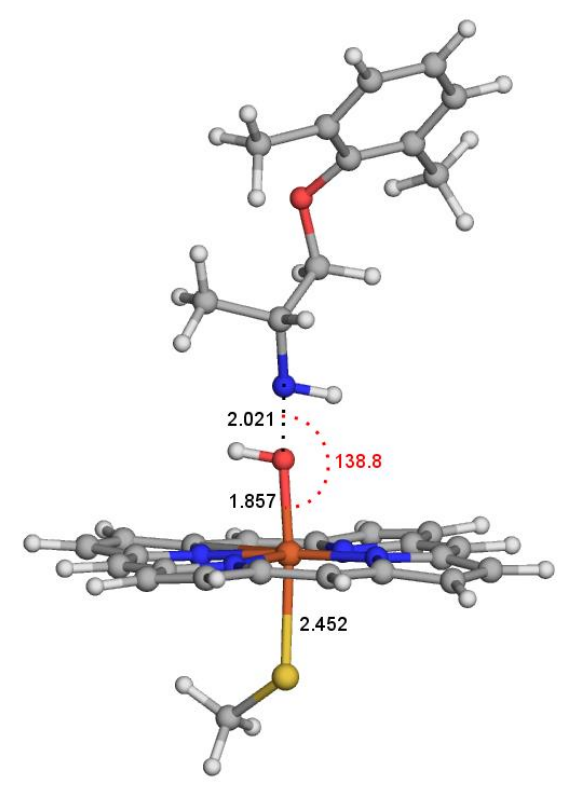


$\mathrm{TS}_{\mathrm{REB}}$ (doublet)

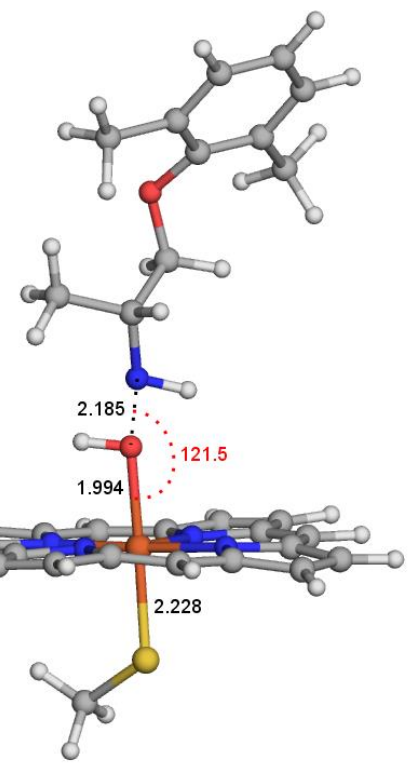

$\mathrm{P}_{\mathrm{REB}}$ (doublet)
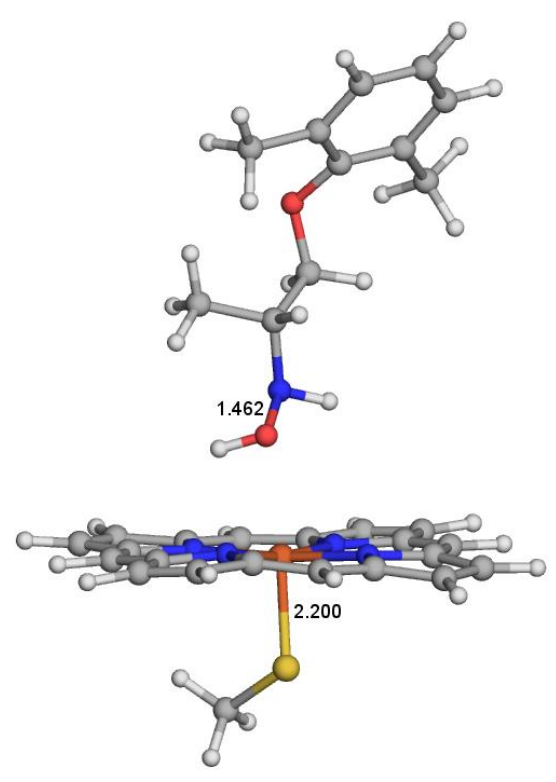

$\mathrm{P}_{\text {REB }}$ (quartet)
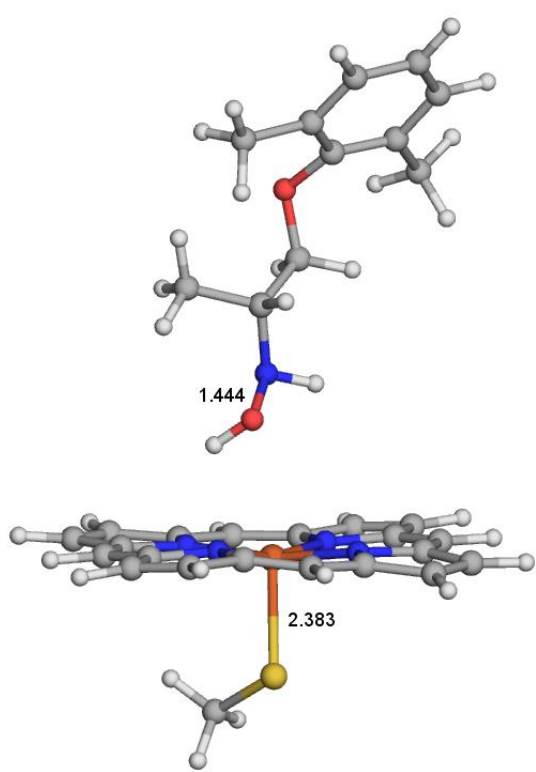